$\mathrm{AB} 0265$

ASSOCIATION OF CUMULATIVE ANTI-CYCLIC CITRULLINATED PROTEIN ANTIBODIES WITH RADIOGRAPHIC PROGRESSION IN PATIENTS WITH RHEUMATOID ARTHRITIS

Young Bin Joo, Yune-Jung Park, Kyung-Su Park, Ki-Jo Kim. Catholic University of Korea, Internal Medicine, Suwon, Korea, Rep. of (South Korea)

Background: Antibody against cyclic citrullinated proteins (ACPA) is counted as one of the most important biomarkers in diagnosis, classification, and prognosis of rheumatoid arthritis (RA). The clinical implications of change in ACPA level over time remain undetermined.

Objectives: We examined the evolution of ACPA during disease course and assess predictive value of time-weighted cumulative ACPA titer on radiographic progression in patients with RA.

Methods: A group of 734 patients with RA was followed longitudinally over 2 years, with annual measurements of $\mathrm{lgM}$ rheumatoid factor (RF) and ACPA. Radiographs of the hands were scored with the modified Sharp score (SHS). Cumulative ACPA antibody titers were calculated using the trapezoidal rule.

Results: The patients with radiographic progression had a higher SHS at baseline; and smoking status, diabetes, RF positivity, and use of biologic DMARDs were independently associated with radiographic progression (all $P<0.05)$. As for ACPA, reversion happened more commonly in men and was associated with younger onset age and lower titer at baseline, but it had no direct relevance to radiographic outcome. In multivariable regression analysis, only high cumulative or baseline titer of ACPA had a predictive power for rapid radiographic progression (all $P<0.05$ ), and cumulative ACPA titer was superior in terms of statistical significance (Cohen's d, 0.637 versus 0.583 ).

Conclusion: High cumulative ACPA titer was an independent predictor for accelerated radiographic progression, especially with initiation of joint damage. Serial measurement of ACPA titer can provide information about its dynamics over the clinical course and can facilitate an additional assessment of radiographic progression.

Acknowledgement: Nothing specified.

Disclosure of Interests: None declared

DOI: 10.1136/annrheumdis-2019-eular.1403

\section{AB0266 THREE NOVEL BIOMARKERS PRDICTING THE SHORT- TERM RESPONSE TO IFX + MTX + LEFTRIPLE THERAPY IN RA}

Jian Chen ${ }^{1}$, Mengshi Tang ${ }^{1}$, Lichang X $\mathrm{u}^{2}$, LI Shu ${ }^{1}, \mathrm{XI} \mathrm{Xie}^{1}$, fen li ${ }^{1}$. ${ }^{1}$ Second Xiangya Hospital of Central South University, Department of Rheumatolog, Changsha, China; ${ }^{2}$ YALI high school, international Department, Changsha, China

Background: For rheumatoid arthritis (RA), nearly one-third of patients still have poor response to biological agents. In addition, infliximab, methotrexate and leflunomide are the most widely used three drugs in the clinic, but there remains no unified and precise indicators capable to predict the clinical response to their combination therapy.

Objectives: The purpose of this experiment is to identify a protein biomarker panel for predicting the outcome of RA patients who have received a triple therapy combining of infliximab, methotrexate and leflunomide (IFX+MTX+LEF).

Methods: All incorporated RA patients with DAS28-CRP $>5.1$ accepted IFX $+M T X+L E F$ therapy. At 14th week, they were divided into good responder (GR), moderate responder (MR), and non-responder (NR) in accordance with the EULAR response criteria. After removal of the 14 high-abundance proteins, serum samples from patients (4 GR and $4 \mathrm{NR}$ ) at baseline and 14th week, and 4 healthy subjects (HS) were screened for candidate biomarkers via isobaric tags for relative and absolute quantification (iTRAQ). After an in-depth technical feasibility analysis, Parallel Reaction Monitoring (PRM) was performed in $20 \mathrm{RA}$ patients and $20 \mathrm{HS}$ for further validation

Results: A total of 590 proteins were identified by ITRAQ, and 51 proteins of which showed significant differences between NR and GR (Figure 1). PRM showed that levels of catalase, epididymal secretory protein $\mathrm{Li}$ 282, and hemoglobin subunit $\delta$ of the GR+MR group at baseline was higher than that of the NR group (Figure 1), and they were involved in the process of oxidative stress.

Conclusion: The RA patients with higher pre-treatment levels of the three proteins responded better to IFX+MTX+LEF triple therapy at 14th week; Inhibitor of oxidative stress might be a new therapeutic target of RA in the future.

\section{REFERENCES}

[1] Tracey D, Klareskog L, Sasso E H, Salfeld J G and Tak P P. Tumor necrosis factor antagonist mechanisms of action: a comprehensive review. Pharmacol Ther, 2008, 117(2):244-279.
[2] Isaacs J D, Ferraccioli G. The need for personalised medicine for rheumatoid arthritis. Ann Rheum Dis, 2011, 70(1):4-7.

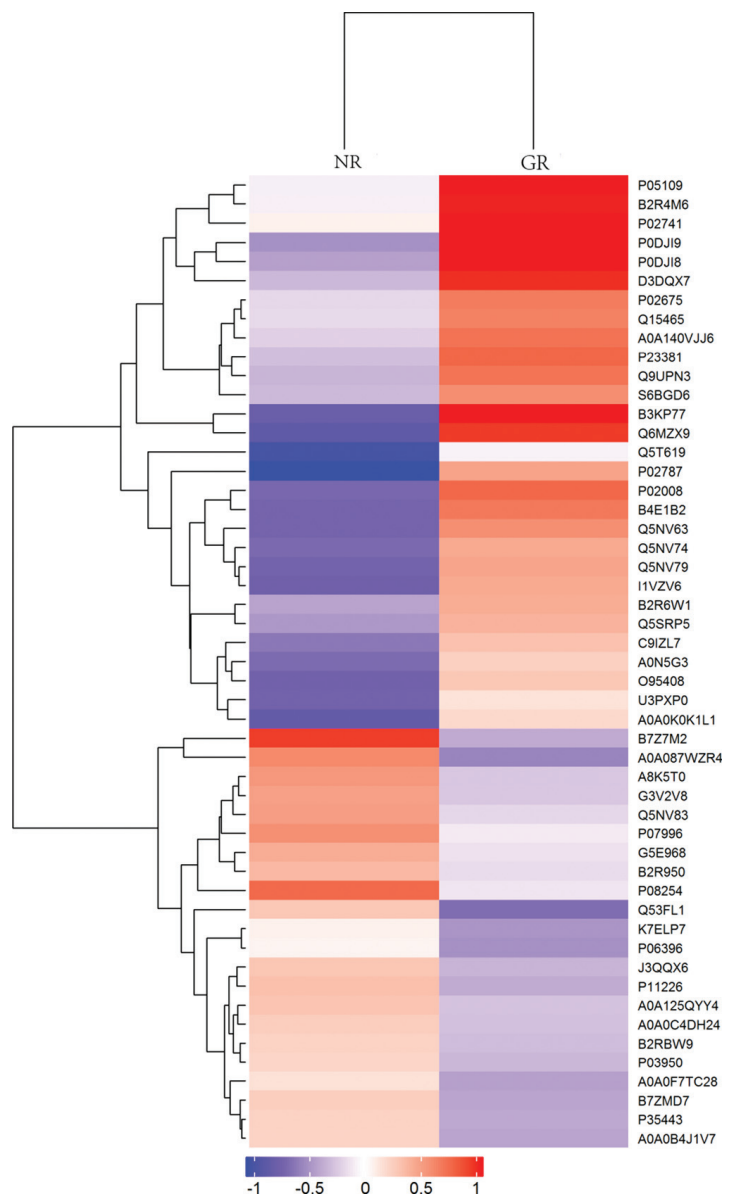

Figure 1. Hierarchical cluster. Hierarchical cluster of proteins differentially expressed between good responders and non-responders with an FDR $<1 \%$ identified by MaxQuan from iTRAQ. Red, high expression; green, low expression. Two main clusters of proteins can be observed, one up-regulated (left) and other down-regulated (right) in the serum of patients. Abbreviation: GR, good responder; NR, non-responder; Red, high expression; Blue, low expression.

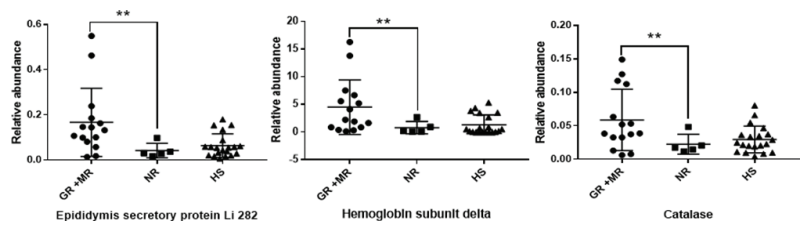

Figure 2. Expression levers of the three protein. Comparison of epididymis secretory protein $\mathrm{Li} 282$, hemoglobin subunit delta and catalase at baseline from PRM among groups of $\mathrm{GR}+\mathrm{MR}, \mathrm{NR}$ and $\mathrm{HS},{ }^{*}{ }^{*} P<0.05$

Disclosure of Interests: None declared DOI: 10.1136/annrheumdis-2019-eular.946

\section{AB0267 ROLE OF FCGAMMA RECEPTORS IIA, IIIA, AND IIIB POLYMORPHISMS IN RHEUMATOID ARTHRITIS SEVERITY}

Ines Mahmoud ${ }^{1}$, Myriam Moalla ${ }^{1}$, Imen Sfar ${ }^{2}$, Olfa Saidane ${ }^{1}$, Aicha Ben Tekaya ${ }^{1}$ Rawdha Tekaya ${ }^{1}$, Elyes Bouajina ${ }^{3}$, Hela Zegaloui ${ }^{3}$, Saloua Aouini ${ }^{2}$, Yousr Gorgi ${ }^{2}$, Leila Abdelmoula ${ }^{1} .{ }^{1}$ Charles Nicolle Hospital, Rheumatology department, Tunis, Tunisia; ${ }^{2}$ Charles Nicolle Hospital, Immunology department, Tunis, Tunisia; ${ }^{3}$ Farhat Hached Hospital, Rheumatology department, Sousse, Tunisia

Background: FC gamma receptors (FcgR) type IIA IIIA and IIIB play an important role in the recognition of immune complexes (ICs) by 
binding their Ig-Fc portion. However, it has been demonstrated that FcgR single nucleotide polymorphisms (SNP) leads to low binding affinity alleles (FcgRIIA-131R, IIIA-158V and IIIB NA2). Therefore, we can make an assumption that the presence of these risk alleles could influence the outcome of immune mediated disease such as rheumatoid arthritis (RA).

Objectives: The aim of this study was to assess the relationship between functional SNP of FcgR IIA H13R, IIIA V158F and IIIB NA1/NA2 and disease severity scores in RA.

Methods: We assessed disease severity in RA patients based on the Health Assessment Questionnaire (HAQ) and Sharp/van der Heijde (mSharp) method. To reduce selection biais, all recruited patients were treated with conventional DMARDs.We considered patients with $\mathrm{HAQ}$ score ranged from 2 to 3 as having severe disability. For radiographic evaluation, since there is not validated threshold for joint damage severity, we took mSharp score median value of our population as a reference. Patients underwent FcgRIIA, FcgRIIIA and FcgRIIIB SNP study using PCR-SSP and direct sequencing process. Then, biallelic polymorphisms were examined for association with $\mathrm{HAQ}$ and mSharp score.

Results: Fifty-eight RA patients were enrolled. The mean duration of the disease was 11.77 years [0.5-24]. Among these patients, 34 (58.62\%) had severe disability according to $\mathrm{HAQ}$ score. The mSharp median value was 43,5 [0-232] and 28 (48.28\%) patients had a score above this value.

Genotypic study showed that FcgR IIIB-NA2 was significantly associated with severe disability $(p=0.021)$ (Table 1$)$.

Table 1. Correlation between FcgR biallelic polymorphisms and disability level

\begin{tabular}{lccccc}
\hline Gene & Genotype & HAQ<2 & HAQ>=2 & OR (95\% Cl) & P-value \\
\hline FcgR IIIB & NA1/NA1 & $10(41.7 \%)$ & $5(14.7 \%)$ & 1.00 & $\mathbf{0 . 0 2 1}$ \\
& NA1/NA2-NA2-NA2 & $\mathbf{1 4}(\mathbf{5 8 . 3} \%)$ & $\mathbf{2 9 ( 8 5 . 3 \% )}$ & $4.14(1.19-14.44)$ & \\
FcgR IIIA & V/V & $4(16.7 \%)$ & $7(20.6 \%)$ & 1.00 & 0.71 \\
& F/F-F/V & $\mathbf{2 0 ( 8 3 . 3 \% )}$ & $\mathbf{2 7 ( 7 9 . 4 \% )}$ & $1.30(0.33-5.04)$ & \\
FcgR IIA & H/H & $3(12.5 \%)$ & $5(14.7 \%)$ & 1.00 & 0.81 \\
& H/R-R/R & $\mathbf{2 1 ( 8 7 . 5 \% )}$ & $\mathbf{2 9 ( 8 5 . 3 \% )}$ & $0.83(0.18-3.86$ & \\
\hline
\end{tabular}

Table 2. Correlation between FcgR biallelic polymorphisms and radiographic evaluation

\begin{tabular}{lccccc}
\hline Gene & Genotype & $\begin{array}{c}\text { Sharp }< \\
\mathbf{4 3 . 5}\end{array}$ & $\begin{array}{c}\text { Sharp } \\
\text { >=43.5 }\end{array}$ & OR (95\% Cl) & $\begin{array}{c}\text { P- } \\
\text { value }\end{array}$ \\
\hline FcgR & NA1/NA1 & $7(23.3 \%)$ & $8(28.6 \%)$ & 1.00 & 0.65 \\
IIIB & NA1/NA2 - NA2- & $23(76.7 \%)$ & $20(71.4 \%)$ & $0.76(0.23-$ & \\
& NA2 & & & $2.47)$ & \\
FcgR & \multirow{2}{*}{ V/V } & $5(16.7 \%)$ & $6(21.4 \%)$ & 1.00 & 0.64 \\
IIIA & F/F-F/N & $25(83.3 \%)$ & $22(78.6 \%)$ & $1.36(0.37-$ & \\
& & & & $5.09)$ & \\
FcgR IIA & H/H & $5(16.7 \%)$ & $3(10.7 \%)$ & 1.00 & 0.51 \\
& H/R-R/R & $25(83.3 \%)$ & $25(89.3 \%)$ & $1.67(0.36-$ & \\
& & & & $7.74)$ & \\
\hline
\end{tabular}

Furthermore, FcgRIIIA-158F and IIA-131R carriers were more frequent in patients with severe disability but the association was not statistically significant. Finally, no correlation was found between radiographic evaluation and FcgR SNP study (table 2).

Conclusion: FcgR low affinity alleles seem to confer susceptibility to severe functional impairment in RA but not to structural damage. However further studies on larger population must be done to corroborate these findings.

\section{REFERENCES}

[1] Brun JG, Madland TM, Vedeler CA. Immunoglobulin G Fc-receptor (FcgammaR) IIA, IIIA, and IIIB polymorphisms related to disease severity in rheumatoid arthritis. J Rheumatol. 2002;29:1135-40.

[2] Kastbom A, Ahmadi A, Soderkvist P, Skogh T. The $158 \mathrm{~V}$ polymorphism of Fc gamma receptor type IIIA in early rheumatoid arthritis: increased susceptibility and severity in male patients (the Swedish TIRA project) Rheumatology (Oxford) 2005;44:1294-8.

[3] Lee YH, Bae SC, Song GG. FCGR2A, FCGR3A, FCGR3B polymorphisms and susceptibility to rheumatoid arthritis: a meta-analysis.Clin Exp Rheumatol. 2015 Sep-Oct; 33 (5):647-54.

Disclosure of Interests: None declared

DOI: 10.1136/annrheumdis-2019-eular.8299

\section{AB0268 \\ MORTALITY RATE OF RHEUMATOID ARTHRITIS PATIENTS EVEN IN THE NEW ERA OF BIOLOGICS IS HIGHER THAN THE CONTROL GROUP: EIGHT YEARS OF THE TOMORROW STUDY}

Koji Mandai ${ }^{1,2}$, Tatsuya Koike ${ }^{1,3}$, Yuko Sugioka ${ }^{3}$, Kentaro Inuii ${ }^{1}$, Tadashi Okano ${ }^{1}$, Yutaro Yamada ${ }^{1}$, Masahiro Tada ${ }^{4}$, Kenji Mamoto ${ }^{5,6}$, Shohei Anno ${ }^{5}$, Hiroaki Nakamura'. ' 'Osaka City University Graduate School of Medicine, Orthopaedic Surgery, Osaka city, Japan; ${ }^{2}$ Osaka social medical center Hospital, Orthopaedic Surgery, OSAKA, Japan; ${ }^{3}$ Osaka City University Medical School, Center for Senile Degenerative Disorders, Osaka city, Japan; ${ }^{4}$ Osaka City General Hospital, Orthopaedic Surgery, Osaka city, Japan; ${ }^{5}$ Yodogawa Christian Hospital, Orthopaedic Surgery, Osaka, Japan; ${ }^{6}$ Clevland clinic, Biomedical Engineering, Program of Advanced Musculoskeletal Imaging (PAMI), Cleveland, United States of America

Background: Patients with rheumatoid arthritis (RA) have a high mortality rate compared to the general population'. However, the mortality rate of patients with RA might be improved by advances in therapy².

Objectives: We investigated the risk factors for mortality in patients with RA from TOMORROW study.

Methods: This study included 413 participants, comprising 208 patients with RA and 205 age- and sex- matched healthy volunteers (Vo) from the prospective "TOMORROW" cohort study that has been ongoing since 2010 were included in this study (women, 84\%; mean age, 58 years old). Median disease duration was 10.3 years.

Results: The rate of accomplishment for 8 years was $83.2 \%$ in the RA group and $92.7 \%$ in the Vo group. There were 14 deaths in the RA group (8.7/1000 person-years) and two in the Vo group (1.2/1000 personyears) $(p=0.0025)$ for 8 years. Infection was the most common cause of death in the RA group (43\%). Cox proportional hazard analysis showed that having RA was significant risk factor for death (hazard ratio [HR]: 6.9, 95\% $\mathrm{Cl}$ : $1.6-30.4, \mathrm{p}=0.01$ ) adjusting by age (Figure). In the RA group, glucocorticoid (GC) use (HR: $3.6,95 \% \mathrm{Cl}: 1.1-10.4, \mathrm{p}=0.032$ ) was significant risk factor for death. Disease activity, duration of disease, use of biological products, use of methotrexate, presence of cardiovascular disease, and smoking were not significant factor.

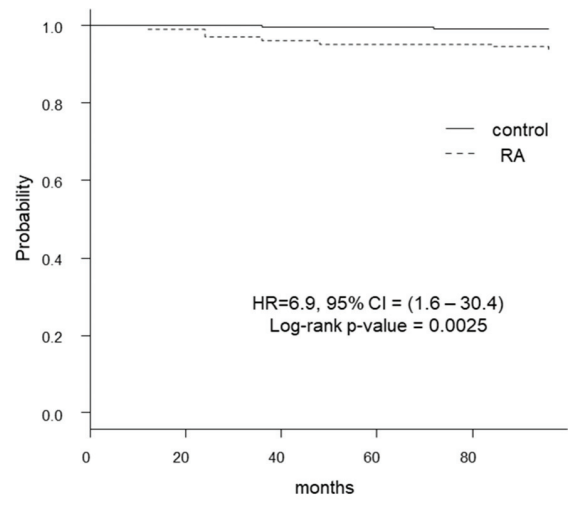

Fig: survival curve of RA and control

Figure 1

Conclusion: RA was still significant risk factor of death. GC use was independent factors of death in RA patients.

\section{REFERENCES}

[1] van den Hoek J, Boshuizen HC, Roorda LD, et al. Mortality in patients with rheumatoid arthritis: a 15-year prospective cohort study. Rheumatol Int (2017) 37:487-493

[2] Lacaille D, Avina-Zubieta JA, Sayre EC, et al. Improvement in five-year mortality in rheumatoid arthritis compared to the general population - closing the mortality gap. Ann Rheum Dis. 2017 June; 76(6): 1057-1063.

Disclosure of Interests: Koji Mandai: None declared, Tatsuya Koike Speakers bureau: AbbVie, Astellas Pharma Inc., Bristol-Myers Squibb, Chugai Pharmaceutical, Eisai, Janssen, Lilly, Mitsubishi Tanabe Pharma Corporation, MSD, Ono Pharmaceutical, Pfizer, Roche, Takeda Pharmaceutical, Teijin Pharma, and UCB, Yuko Sugioka: None declared, Kentaro Inui Speakers bureau: Takeda Pharmaceutical, Pfizer Japan, Daiichi-Sankyo Co.Ltd., Abbvie, Mitsubishi Tanabe Pharma Corporation, Janssen Pharmaceutical, Chugai Pharmaceutical, Ono Pharmaceutical, Eisai Co. Ltd., Eli-Lilly, Nippon Kayaku Co.,Ltd., Maruho Co.,Ltd, Kaken 